\title{
microRNA-10b is a prognostic biomarker for melanoma
}

\author{
Gerald Saldanha ${ }^{1,2}$, Shona Elshaw ${ }^{1}$, Parysatis Sachs ${ }^{1}$, Hisham Alharbi ${ }^{1}$, Prashant Shah ${ }^{1}$, \\ Ann Jothi ${ }^{1}$ and J Howard Pringle ${ }^{1}$ \\ ${ }^{1}$ Department of Cancer Studies, University of Leicester, Leicester, UK and ${ }^{2}$ EMPATH, University Hospitals of \\ Leicester, Leicester, UK
}

\begin{abstract}
Malignant melanoma is an aggressive form of skin cancer. Recently, drug therapy of advanced disease has been revolutionized by new agents. More therapeutic options, coupled with the desire to extend treatment to the adjuvant setting mean that prognostic biomarkers that can be assayed from formalin-fixed paraffin-embedded clinical would be valuable. microRNAs have potential to fill this need. We analyzed 377 microRNAs in 79 primary melanomas and $\mathbf{3 2}$ metastases using a split sample discovery strategy. From a discovery analysis using $\mathbf{4 0}$ thick primary melanomas (20 cases with metastasis and 20 controls without metastasis at 5 years), microRNA expression was measured by quantitative RT-PCR (QRT-PCR). MiR-10b emerged as a candidate prognostic microRNA. This was confirmed in an independent validation set of thick primary melanomas (20 cases with metastasis and 19 controls without metastasis at 5 years). In the combined discovery and validation cohorts $(n=79)$, miR-10b expression showed a 3.7-fold increase in expression between cases and controls $(P=0.005)$ and showed a trend of increasing expression between primary melanomas and their matched metastases $(P<0.001)$. In situ hybridization showed expression was in melanoma cells and correlated with expression measured by QRT-PCR $(P=0.0005)$. We used the combined discovery and validation samples to verify the prognostic value of additional candidate microRNAs identified from other studies, and proceeded to analyze miR-200b. We demonstrated that miR-10b and miR-200b showed independent prognostic value $(P=0.002$ and 0.047 , respectively) in multivariable analysis alongside known clinico-pathological prognostic features (eg, Breslow thickness) using a Cox proportional hazards regression model. Furthermore, the addition of these microRNAs to the clinico-pathological features led to an improved regression model with better identification of aggressive thick melanomas. Taken together, these data suggest that miR-10b is a new prognostic microRNA for melanoma and that there could be a place for microRNA analysis in stratifying melanoma for therapy. Modern Pathology (2016) 29, 112-121; doi:10.1038/modpathol.2015.149; published online 8 January 2016
\end{abstract}

Melanoma is an aggressive skin cancer. Most tumors can be cured by local surgical excision but once spread to distant sites occurs the prognosis is poor. However, recent clinical trials have provided renewed hope for patients with advanced melanoma. These new therapeutic avenues mean that a search for tissue biomarkers is very timely, especially prognostic biomarkers that can stratify patients into subgroups with varying therapeutic need.

One of the major problems in finding relevant biomarkers is that macromolecules are poorly preserved in clinical melanoma samples because all of the tumor tissue is typically fixed in formalin and then paraffin embedded. However, microRNAs

Correspondence: Dr G Saldanha, University of Leicester, Level 3 Robert Kilpatrick Building, Leicester Royal Infirmary, Leicester LE2 7LX, UK. E-mail: gss4@le.ac.uk

Received 8 June 2015; accepted 16 November 2015; published online 8 January 2016
(miRNAs) have attracted attention because they are reasonably well preserved. These are small, non-coding single-stranded RNAs that are about 22 nucleotides in length and that function post transcriptionally as negative regulators of gene expression. ${ }^{1}$ Altered miRNA expression in cancer was first reported in 2002, with downregulation of miR-15 and miR-16 expression, which target B-cell lymphoma 2 (BCL2) in chronic lymphocytic leukemia. ${ }^{2}$ Many subsequent reports of miRNAs in cancer have been reviewed. ${ }^{3}$

In melanoma, miRNAs can be readily analyzed from formalin-fixed paraffin-embedded tissue, ${ }^{4}$ show high frequency genetic alterations, ${ }^{5}$ and exhibit a melanoma-specific signature. ${ }^{6}$ In addition, miRNAs have shown prognostic value in melanoma. ${ }^{7-11}$ A panel of six miRNAs was identified as a post-recurrence survival signature, ${ }^{9}$ and miRNA and mRNA networks were associated with the 


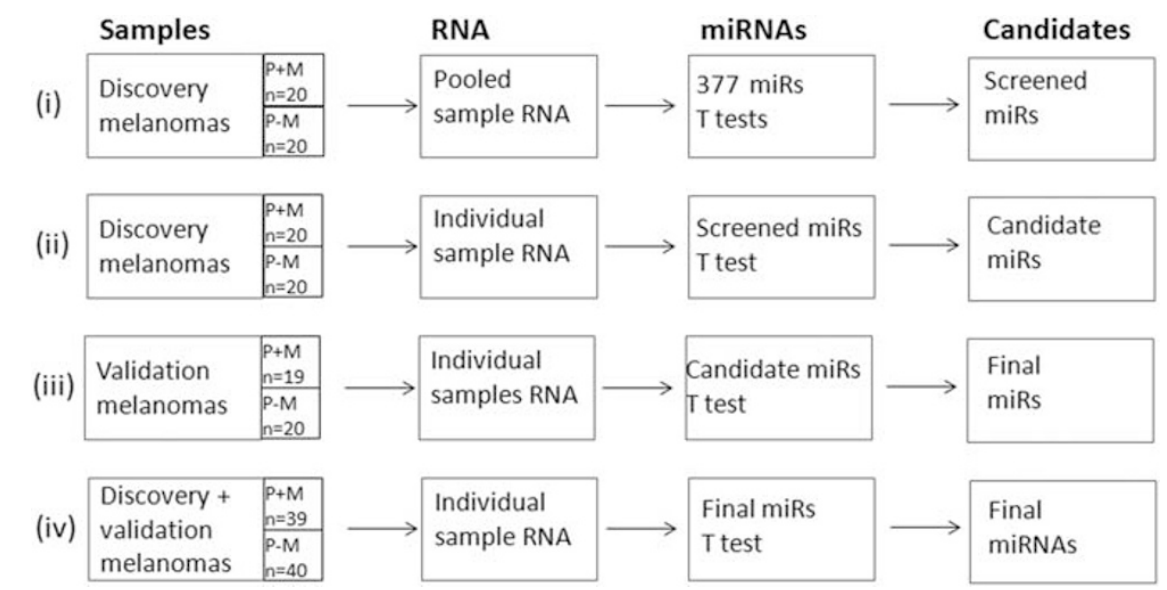

Figure 1 Workflow for discovery of microRNAs. P+M, primary melanoma that metastasized within 5 years; P - M, primary melanoma with no metastases within 5 years.

outcome, ${ }^{11}$ while a 4 -miRNA signature was associated with brain metastasis. ${ }^{12}$ Several anti-miRNA agents are under development ${ }^{13}$ and therefore tissue miRNA analysis has potential predictive value in the future.

The evidence thus far shows that miRNAs are well preserved in formalin-fixed paraffin-embedded melanoma tissue and that they have a potential to be prognostic biomarkers. Therefore we sought to identify miRNAs with prognostic value and to determine whether they could provide independent prognostic significance beyond routine clinical and pathological factors used in clinical staging, focusing on at risk patients with thicker melanomas. To test the hypothesis that miRNAs have prognostic value we compared differential expression of 377 miRNAs in non-metastasizing and metastasizing primary melanomas using a four-step approach based on split-sample methodology. In addition, we also sought to validate a small number of prognostic miRNAs already identified by others in previous studies.

\section{Materials and methods}

\section{Patients and Setting}

To discover candidate prognostic miRNAs in melanoma tissue we compared non-metastasizing and metastasizing primary melanomas at high risk of progression (ie, mainly $>2 \mathrm{~mm}$ thick). The focus on thicker melanomas is relevant because these are the tumors most likely to metastasize and kill. We used a split-sample methodology comprising discovery and validation sets, where each set contained controls and cases. The discovery set comprised 'controls' that consisted of 20 primary melanomas that were metastasis-free for at least 5 years $(P-M)$; the 'cases' were tissues from 20 primary melanomas that had developed metastasis within 5 years
$(\mathrm{P}+\mathrm{M})$. For 16 of the $\mathrm{P}+\mathrm{M}$ cases, we also analyzed tissue from the first resected matched metastasis (Mets). The validation set was similarly assembled, comprising 20P-M controls, 20P+M cases (one failed to have sufficient RNA yield, so final number was 19) and 16 Mets, 10 of which were matched to the $\mathrm{P}+\mathrm{M}$ cases. All melanomas samples were selected from the pathology archives of the University Hospitals of Leicester NHS Trust. The $40 \mathrm{P}+\mathrm{Ms}$ were AJCC7 stage IIA, IIB, or IIC at diagnosis. On subsequent review during data analysis, two of the $\mathrm{P}+\mathrm{M}$ melanomas turned out to be stage IB. The $40 \mathrm{P}-\mathrm{Ms}$ were AJCC7 stage IIA, IIB, or IIC. Because of difficulty in identifying sufficient numbers of $\mathrm{P}-\mathrm{M}$ cases, we also included one that was stage IB.

The discovery strategy entailed four steps: (i) an initial screen of 377 miRNAs, analyzing pooled RNA from the discovery set of melanomas; (ii) testing of screened miRNAs in individual (ie, not pooled) discovery set melanomas to yield candidates; (iii) confirmation of miRNA candidates in the independent validation set of melanomas to yield final miRNAs; (iv) final analysis on combined discovery and validation sets of melanomas. These steps are summarized in Figure 1. In the first step, the discovery set was made into eight pooled cDNA samples, four pools of $\mathrm{P}-\mathrm{M}$ and four pools of $\mathrm{P}+\mathrm{M}$, with each pool containing five melanomas (Table 1). Pools 1-4 were approximately in ascending AJCC ' $\mathrm{T}$ ' stage order. The matched metastases were made into four pools of four cases, such that metastasis pool 1 contained DNA matched to that from four of the cases in $\mathrm{P}+\mathrm{M}$ pool 1 and similarly for subsequent pools. In addition to the four steps above, we proceeded to use the full set of melanoma samples to validate a small number of miRNAs that had been identified as candidates for having prognostic relevance to melanoma from previous publications. Given the large number of candidate miRNAs in the published literature, we focused on a small 
Table 1 Pooled discovery primary melanoma sample characteristics

\begin{tabular}{|c|c|c|c|}
\hline Controls pool 1 & & Cases pool 1 & \\
\hline Average age & Average Breslow depth & Average age & Average Breslow depth \\
\hline 66 & 1.9 & 60 & 2.8 \\
\hline Median age & Median Breslow depth & Median age & Median Breslow depth \\
\hline 68 & 2.0 & 57 & 2.5 \\
\hline Controls pool 2 & & Cases pool 2 & \\
\hline Average age & Average Breslow depth & Average age & Average Breslow depth \\
\hline 77 & 3.3 & 66 & 3.2 \\
\hline Median age & Median Breslow depth & Median age & Median Breslow depth \\
\hline 74 & 3.2 & 63 & 3.5 \\
\hline Controls pool 3 & & Cases pool 3 & \\
\hline Average age & Average Breslow depth & Average age & Average Breslow depth \\
\hline 63 & 3.1 & 61 & 5.3 \\
\hline Median age & Median Breslow depth & Median age & Median Breslow depth \\
\hline 68 & 2.9 & 58 & 4.5 \\
\hline Controls pool 4 & & Cases pool 4 & \\
\hline Average age & Average Breslow depth & Average age & Average Breslow depth \\
\hline 74 & 7.6 & 72 & 8.2 \\
\hline Median age & Median Breslow depth & Median age & Median Breslow depth \\
\hline 75 & 7.4 & 71 & 9.7 \\
\hline
\end{tabular}

group of candidates that were relevant to studies published from our own laboratories. Specifically, we assessed miR-21 because we have previously analyzed this in blood ${ }^{14}$ and other groups have found it to be relevant to melanoma, ${ }^{15-19}$ and the miR200 family and mir205 because they influence EMT master regulators, about which $\mathrm{we}^{20}$ and others $^{21-26}$ have previously published. Because these miRNAs had been previously discovered as melanoma prognostic biomarker candidates in independent experiments with different case sets, we combined the training and validation sets from the current study, and then analyzed these miRNAs in individual samples.

\section{Tissue Micro-Dissection, Lysates, and Total RNA Preparation}

Manual micro-dissection was performed. Sections were de-waxed and digested in $500 \mu \mathrm{l}$ of lysis buffer (0.05 M Tris, pH 7.65, 0.1\% SDS, and $100 \mu \mathrm{g} / \mathrm{ml}$ proteinase K overnight at $37^{\circ} \mathrm{C}$ ). Lysates were stored at $-20^{\circ} \mathrm{C}$. Total RNA was purified from tissue digests by Tri-reagent (Sigma-Aldrich, Gillingham, UK) separation using the manufacturer's protocol for tissue or plasma. Once separated, the total RNA was precipitated with 1.25 times the volume of absolute ethanol using the Qiagen RNeasy kit (Qiagen, Valencia, CA, USA) or the mirVana miRNA (Life Technologies Ltd, Paisley, UK) isolation kit following the manufacturer's protocols. The RNA was extracted in $25 \mu \mathrm{l}$ of RNase-free buffer and stored at $-20^{\circ} \mathrm{C}$.

\section{Reverse Transcription and Pre-Amplification}

miRNAs were profiled using a two-step process. Initially, cDNA was synthesized from total RNA using a Taqman microRNA Reverse Transcription Kit and Taqman Megaplex RT primers (Human Pool A v2.0) in a total of $7.5 \mu \mathrm{l}$ volume (Applied Biosystems, Foster City, CA, USA). The cDNA produced by the RT reaction was then pre-amplified to improve sensitivity and increase the number of detectable miRNAs using Taqman Megaplex Pre-Amplification Primers (Human Pool A v2.0) and Taqman Pre-Amplification Master Mix in a $25 \mu \mathrm{l}$ reaction according to the manufacturer's instructions. Pre-amplified cDNA was diluted with $0.1 \times$ TE (pH 8.0) to $100 \mu \mathrm{l}$.

\section{Assessment of miRNA Expression in Discovery Cohort from Pooled cDNA}

Pooled microRNAs were assessed using microfluidic cards. We used the Taqman stem-loop QRT-PCRbased array method (Taqman microRNA array A card v2.0) representing 377 highly characterized miRNAs present in the Sanger miRBase v12. Each array card contained three positive controls (U6 snRNA, RNU44, and RNU48) and a negative control (ath-miR-159a). QRT-PCR was performed using Applied Biosystems 7900HT system and a Taqman Universal PCR mastermix with $9 \mu \mathrm{l}$ cDNA input per plate. The expression levels of miRNAs in all groups were calculated using the comparative cycle threshold $\left(\mathrm{C}_{\mathrm{T}}\right)$ method, whereby a lower $\mathrm{C}_{\mathrm{T}}$ value is consistent with faster detection of fluorescence and therefore higher expression, and SDS software v2.3 using automatic baseline settings and a threshold of 0.2. Samples were normalized by the mean of expressed miRNAs, as described, ${ }^{27}$ whereby $\mathrm{C}_{\mathrm{T}}$ values $\geq 35$ were considered to be below the detection level of the assay and therefore only miRNAs with a $\mathrm{C}_{\mathrm{T}}$ value $<35$ were included in the analyses. The mean miRNA $\mathrm{C}_{\mathrm{T}}$ value was then calculated for each group and subtracted from each 
individual miRNA value to give a $\Delta \mathrm{C}_{\mathrm{T}}$ for each sample. Fold changes in miRNA expression were calculated using the equation $R Q=2^{-\Delta \Delta C_{T}}$ as described, ${ }^{28}$ where comparative $\mathrm{C}_{\mathrm{T}}\left(\Delta \Delta \mathrm{C}_{\mathrm{T}}\right)$ is defined as the difference between $\Delta \mathrm{C}_{\mathrm{T}}$ of comparison groups (eg, $\left.\left(\Delta \mathrm{C}_{\mathrm{T}} \mathrm{P}+\mathrm{M}\right)-\left(\Delta \mathrm{C}_{\mathrm{T}} \mathrm{P}-\mathrm{M}\right)\right)$. miRNAs were ranked according to the $P$-value based on a $t$-test comparing mean $\Delta \mathrm{C}_{\mathrm{T}}$ of the four $\mathrm{P}-\mathrm{M}$ and four $\mathrm{P}+\mathrm{M}$ pools. These were further filtered by fold change to identify miRNAs with the biggest effect size (filter $>2.5$-fold change), and by level of expression across all pools to ensure cases with barely detectable expression were excluded (filter $=\Delta \mathrm{C}_{\mathrm{T}}<0$ ). We also documented as a secondary analysis a $t$-test for $\Delta \mathrm{C}_{\mathrm{T}}$ values of $\mathrm{P}+\mathrm{M}$ vs Metastases.

\section{Verification of miRNA Expression in Single Sample cDNA}

microRNA expression in single cDNA samples was quantified using the stem-loop real-time PCR method $^{28}$ and Taqman microRNA individual assays (Applied Biosystems). All reactions, including no-template controls and -RT controls were performed using a 96-well StepOnePlus Fast Real-Time PCR system (Applied Biosystems). Cycle thresholds were normalized to three endogenous control miRNAs (miR-191, miR-345, and miR-132) that were identified by Genorm ${ }^{29}$ and Normfinder ${ }^{30}$ as being most stably representative of the mean of expressed miRNAs from the Taqman microRNA array data described. ${ }^{29}$ The average $C_{T}$ value of endogenous miRNAs was subtracted from the $\mathrm{C}_{\mathrm{T}}$ value for each individual miRNA reaction, resulting in the $\Delta \mathrm{C}_{\mathrm{T}}$ value.

\section{In Situ Hybridization for miR-10b}

We wanted to assess whether C-ISH could eventually be used in future studies because this is more accessible to clinical histopathology laboratories and an assay that preserves morphology would be better understood by histopathologists, the gatekeepers for testing on clinical tissue samples. We designed C-ISH probes and used a semi-quantitative scoring system to assess miRNA expression. This was then compared with the expression as determined by stem-loop QRT-PCR in 13 cases with sufficient tissue sections for analysis. In situ hybridization for miR-10b was based on a described method ${ }^{31}$ using $6 \mu \mathrm{m}$ thick formalin-fixed, paraffin-embedded sections. These were de-waxed, rehydrated, and digested with proteinase $\mathrm{K}$ at $7.5 \mu \mathrm{g} / \mathrm{ml}$ in $50 \mathrm{mM}$ Tris- $\mathrm{HCl} \mathrm{pH} 7.6$ at $37^{\circ} \mathrm{C}$ for $30 \mathrm{~min}$, then pre-hybridized in Exiqon hybridization buffer (Exiqon, Vedbaek, Denmark) at $56^{\circ} \mathrm{C}$ for $15 \mathrm{~min}$ and hybridized for $2 \mathrm{~h}$ with $80 \mathrm{nM}$ double-DIG-labeled miR-10b probe with 30\% Locked Nucleic Acid (LNA) substitutions complimentary to full-length miR-10b $\mathrm{CA}+\mathrm{CAA}+\mathrm{ATT}+\mathrm{CGG}+\mathrm{TTC}$
+TAC+AGG+GTA (base after+is locked) (Exiqon), given stringent washes with $5 \times$ SSC, $1 \times$ SSC, and $0.2 \times$ SSC buffers at $55^{\circ} \mathrm{C}$ for $5 \mathrm{~min}$ per wash, blocked with DIG blocking reagent (Roche, Mannheim, Germany) in maleic acid buffer containing 2\% sheep serum at $25^{\circ} \mathrm{C}$ for $15 \mathrm{~min}$, and detected with alkaline phosphatase-conjugated anti-digoxigenin (1:600 in blocking reagent, Roche) for $15 \mathrm{~min}$, enzymatic development using 4-nitro-blue tetrazolium (NBT) and 5-brom-4-chloro-3'-Indolylphosphate (BCIP) substrate (Roche) at $25^{\circ} \mathrm{C}$ for $180 \mathrm{~min}$. A nuclear fast red counterstain (Vector Laboratories, Burlingname, CA, USA), at $25^{\circ} \mathrm{C}$ for $2 \mathrm{~min}$, was used. The slides were mounted with Eukitt mounting medium (VWR, Herlev, Denmark). Negative controls were carried out using scrambled probe $(80 \mathrm{nM})$ or the scrambled probe double-DIG-labeled scramble probe (Exiqon, Cat \#99004-15) and by omission of the probe in the hybridization protocol. A double-DIG-labeled probe for U6 snRNA (cacgaatttgcgtgtcatcctt, Exiqon) $(0.1 \mathrm{nM})$ was used as a positive contol to test for RNA retention. Four photographs were taken of each tissue section. Each of these images was analyzed with the ImageScope software, which was set to categorize staining intensity into weak positive, positive, and strong positive. A ' $\mathrm{H}$ Score' was calculated by multiplying the weak positive, positive, and strong positive proportions by 1,2 , and 3 , respectively, to yield a final score between 0 and 300. The mean score of four fields was used for each case.

\section{Statistical Methods}

$T$-tests were used to compare miRNAs between cases $(\mathrm{P}+\mathrm{M})$ and controls $(\mathrm{P}-\mathrm{M})$ for 377 miRNAs. The Benjamini and Hochberg false discovery rate was used $^{32}$ for multiple testing correction. Multiple groups were compared using ANOVA and matched samples with a paired $t$-test. Prognostic value of miRNAs was assessed using logistic regression. We looked at whether miRNAs could be used to add prognostic value to the established clinical prognostic factors and assessed this by fitting a logistic regression model to the data from the $\mathrm{P}-\mathrm{M}$ and $\mathrm{P}+\mathrm{M}$ primary melanomas from the above discovery and validation sets $(n=79)$. The outcome variable was 5-year metastasis status. Initial univariate logistic regression models were fitted. Next, we created a two-step model, first using as covariates clinicopathological prognostic variables for which we possessed data (age, gender, site, Breslow depth, and ulceration) and then, in step 2, adding in candidate miRNAs to assess whether they improved model accuracy and prediction. Pearson's correlation was used to assess ISH and QRT-PCR correlation. Alpha was set at 0.05 and two-tailed tests were used. All analyses were performed using Microsoft Excel 2010, Graphpad Prism 6.0 and IBM SPSS version 20. 
Table 2 Discovery and validation case clinico-pathological features

\begin{tabular}{|c|c|c|c|c|}
\hline & \multicolumn{2}{|c|}{ Discovery samples } & \multicolumn{2}{|c|}{ Validation samples } \\
\hline & $P-M(\mathrm{n}=20)$ & $P+M(\mathrm{n}=20)$ & $P-M(\mathrm{n}=20)$ & $P+M(\mathrm{n}=19)$ \\
\hline \multicolumn{5}{|l|}{ Stage (AJCC7) } \\
\hline IB & 0 & 0 & $1(5 \%)$ & $2(10 \%)$ \\
\hline IIA & $11(55 \%)$ & $6(30 \%)$ & $15(75 \%)$ & $5(26 \%)$ \\
\hline IIB & $8(40 \%)$ & $9(45 \%)$ & $2(10 \%)$ & $6(32 \%)$ \\
\hline IIC & $1(5 \%)$ & $5(30 \%)$ & $2(10 \%)$ & $6(32 \%)$ \\
\hline \multicolumn{5}{|l|}{ Breslow } \\
\hline Mean (mm) & 3.7 & 4.8 & 3.0 & 4.6 \\
\hline Median (mm) & 3.0 & 4.0 & 2.5 & 3.4 \\
\hline \multicolumn{5}{|l|}{ Age } \\
\hline Mean & 68 & 65 & 62 & 63 \\
\hline Median & 68 & 61.5 & 63 & 63 \\
\hline \multicolumn{5}{|l|}{ Gender } \\
\hline Male & $5(25 \%)$ & $11(55 \%)$ & $11(55 \%)$ & $11(58 \%)$ \\
\hline \multicolumn{5}{|l|}{ Site } \\
\hline Arm & $4(20 \%)$ & $3(15 \%)$ & $5(25 \%)$ & $3(16 \%)$ \\
\hline Leg & $7(35 \%)$ & $9(45 \%)$ & $6(30 \%)$ & $6(32 \%)$ \\
\hline Trunk & $3(15 \%)$ & $5(25 \%)$ & $6(30 \%)$ & $7(37 \%)$ \\
\hline Head and neck & $6(30 \%)$ & $3(15 \%)$ & $2(10 \%)$ & $1(5 \%)$ \\
\hline Unknown & 0 & 0 & $1(5 \%)$ & $2(10 \%)$ \\
\hline
\end{tabular}

Table 3 Candidate miRNAs from comparisons of pooled RNA samples

\begin{tabular}{|c|c|c|c|c|c|}
\hline & $\begin{array}{c}P-M \text { vs } P+M \\
(\mathrm{P} \text {-value) }\end{array}$ & $\begin{array}{c}P+M \text { vs mets } \\
\text { (P-value) }\end{array}$ & $\begin{array}{l}P-M \text { vs } P+M \\
\text { (fold change) }\end{array}$ & $\begin{array}{l}P+M \text { vs met } \\
\text { (fold change) }\end{array}$ & $\begin{array}{c}\text { Absolute expression } \\
\left.\text { (mean } \Delta C_{T}\right)\end{array}$ \\
\hline hsa-miR-10b & 0.0158 & 0.0468 & 4.19 & 4.70 & -1.17 \\
\hline hsa-miR-137 & 0.0207 & 0.1064 & 0.02 & 14.48 & 9.52 \\
\hline hsa-miR-19b & 0.0229 & 0.5761 & 1.50 & 1.14 & -3.63 \\
\hline hsa-miR-548c-3p & 0.0253 & 0.1808 & 0.06 & 4.86 & 10.46 \\
\hline hsa-miR-126 & 0.0292 & 0.1499 & 1.57 & 1.49 & -4.69 \\
\hline hsa-miR-523 & 0.0342 & 0.0963 & 0.27 & 0.20 & 6.94 \\
\hline hsa-miR-886-5p & 0.0364 & 0.1600 & 2.21 & 0.69 & -5.74 \\
\hline hsa-miR-885-5p & 0.0497 & 0.0450 & 0.32 & 0.42 & 1.52 \\
\hline hsa-miR-328 & 0.0728 & 0.1146 & 0.73 & 0.77 & -4.00 \\
\hline hsa-miR-517a & 0.0766 & 0.9761 & 0.10 & 0.95 & 6.38 \\
\hline sa-miR-521 & 0.0786 & 0.7788 & 0.25 & 0.86 & 11.60 \\
\hline hsa-miR-222 & 0.0948 & 0.2039 & 0.69 & 1.31 & -8.48 \\
\hline hsa-miR-494 & 0.0967 & 0.6861 & 1.40 & 0.95 & -2.13 \\
\hline hsa-miR-33b & 0.0976 & 0.3236 & 0.13 & 2.24 & 11.22 \\
\hline hsa-miR-489 & 0.1079 & 0.8914 & 2.20 & 1.05 & 1.72 \\
\hline hsa-miR-372 & 0.1156 & 0.5979 & 10.70 & 0.55 & 9.22 \\
\hline hsa-miR-518f & 0.1182 & 0.1182 & 0.26 & 3.63 & 5.58 \\
\hline hsa-miR-380 & 0.1202 & 0.4516 & 0.30 & 2.29 & 11.62 \\
\hline hsa-miR-219-5p & 0.1207 & 0.2651 & 0.09 & 2.74 & 10.94 \\
\hline
\end{tabular}

Grayed cell means criterion was satisfied.

\section{Results}

\section{Discovery of Candidate Prognostic miRNAs}

The clinico-pathological features of the primary melanomas are shown in Table 2.

The top 10 miRNAs ranked on $P$-value with other filter criteria are shown in Table 3 . None of the
miRNAs passed a FDR filter of $5 \%$, perhaps because this data was derived from pooled rather than individual samples. Because of this, further analysis was limited to the top ranked candidate only, miR-10b. Additional reassurance for further analysis of this miRNA was provided by the fact that all other filter criteria were met (see shaded cells in Table 3). 
a

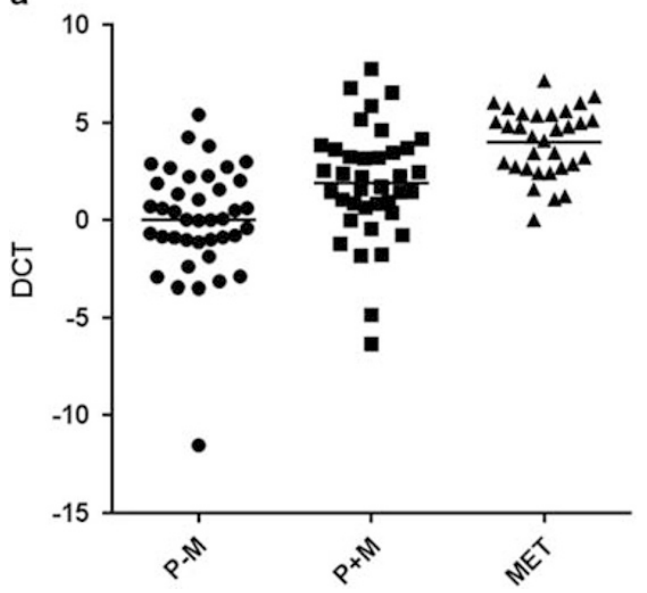

b

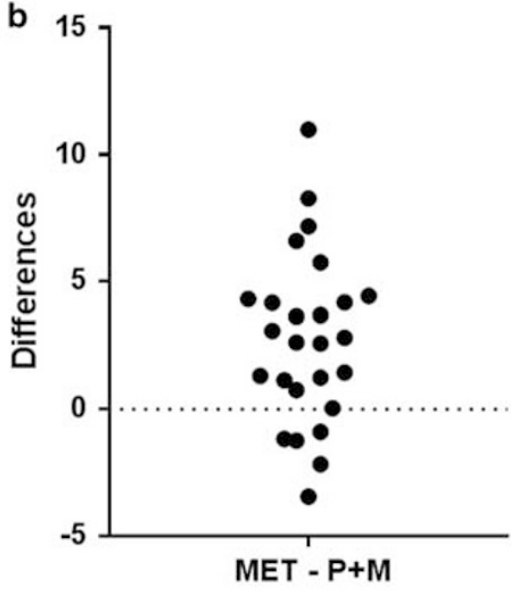

Figure 2 MiR-10b expression in primary melanoma controls, cases, and metastases. (a) Controls ( $\mathrm{P}-\mathrm{M})$, cases ( $\mathrm{P}+\mathrm{M})$, and metastases showed a significant trend of increasing miR-10b expression $(P<0.0001)$. (b) Cases and matched metastases showed a significant increase in miR-10b expression $(P<0.001)$. DCT, delta CT; $\mathrm{P}+\mathrm{M}$, melanoma with metastasis within 5 years; Met $-(\mathrm{P}+\mathrm{M})$, difference in paired metastasis and $\mathrm{P}+\mathrm{M}$ delta CT.

We identified miR-191, miR-345, and miR-132 as the most stably expressed from the 377 in the TaqMan array and these were used to normalize individual samples. The mean difference in $\Delta \mathrm{C}_{\mathrm{T}}$ between $20 \mathrm{P}-\mathrm{M}$ controls and $20 \mathrm{P}+\mathrm{M}$ cases was $-1.38(95 \%$ CI -2.69 to -0.07$), P$-value 0.04 . This was in keeping with a 2.6-fold rise in miR10b expression in $\mathrm{P}+\mathrm{M}$ samples. In order to confirm these findings, and particularly because miR-10b did not pass the FDR threshold in step $\mathrm{i}$, an entirely independent set of $20 \mathrm{P}-\mathrm{M}$ controls and $19 \mathrm{P}+\mathrm{M}$ cases was assessed in step iii of the analysis. The difference in $\Delta \mathrm{C}_{\mathrm{T}}$ was -2.39 (95\% CI -4.69 to -0.10$)$, indicating a 5.2 -fold increase in $\mathrm{P}+\mathrm{M}$ expression, $P$-value 0.04. Significant $P$-values in two independent sets of samples support the validity of miR10b as a potential biomarker for detecting thicker melanomas at higher risk of metastasis. In step iv of the analysis, the discovery and validation sets were combined to assess additional statistical parameters. This set comprised 111 samples (40P-M, 39P+M, and 32 metastases). $\mathrm{P}-\mathrm{M}$ and $\mathrm{P}+\mathrm{M}$ had mean mir-10b $\Delta \mathrm{C}_{\mathrm{T}}$ values of 0.00 and -1.88 , respectively, with a difference of $1.88 \quad(95 \%$ CI $0.59-3.16, t=2.91, \mathrm{df}=77, P=0.0047$ ), indicating a fold change of 3.7 (Figure 2a). An ANOVA comparing $\mathrm{P}-\mathrm{M}, \mathrm{P}+\mathrm{M}$, and metastases (latter, mean $\Delta \mathrm{C}_{\mathrm{T}}-4.00$ ) showed a significant difference between groups $(P<0.0001, F=21.25, \mathrm{df}=108,2)$ with a significant positive linear trend $(P<0.0001)$. The $\mathrm{P}+\mathrm{M}$ and their matched metastases ( $n=26$ pairs) shows a rise in expression in metastases from $\Delta \mathrm{C}_{\mathrm{T}}-1.21(\mathrm{P}+\mathrm{M})$ to -3.95 (Mets), fold change 6.7, $P<0.001$ (Figure 2b).

\section{miR10b C-ISH Correlates with QRT-PCR}

C-ISH was performed on 13 melanoma cases using a probe against miR-10b. This demonstrated that mir-10b was present in melanoma cells and not stromal cells and revealed a strong correlation between miR-10b expression measured by C-ISH and by QRT-PCR. Pearson's correlation coefficient was $0.83(P=0.0005)$. This relationship, alongside representative C-ISH photomicrographs, is shown in Figure 3.

\section{Validation of miRNAs from Other Studies}

Analysis of miR-21, miR-200 family, and miR-205 was performed and the results are shown in Table 4. This corroborates the prognostic relevance of some of these miRNAs. MiR-200b had the largest effect size and lowest $P$-value and was therefore used alongside miR-10b for further analysis below.

\section{Prognostic Value of miRNAs}

Both miR-10b and miR-200b were significant in univariable Cox proportional hazards regression (miR-10b: $\quad P=0.009, \quad$ OR 1.29, CI 1.07-1.57; miR-200b: $P=0.024$, OR 0.78, CI 0.62-0.97). In multivariable analysis featuring known prognostic predictors, only Breslow thickness was significant $(P=0.021$, OR 1.34, CI 1.05-1.72). When the two miRNAs were added to the regression model, the only variables that remained significant were miR10b $(P=0.002$, OR $1.55,95 \%$ CI $1.17-2.06)$ and miR200b $(P=0.047$, OR 0.78, CI 0.61-0.997; Table 5). Most importantly, addition of miR-10b and miR-200b to the model improved classification of $\mathrm{P}-\mathrm{M}$ and $\mathrm{P}+\mathrm{M}$ from $65.8 \%$ (52/79) to $72.2 \%$ (57/79), with a reduction in model Chi-squared (17.69, df 2 , $P<0.001)$ when compared with clinico-pathological variables alone. 

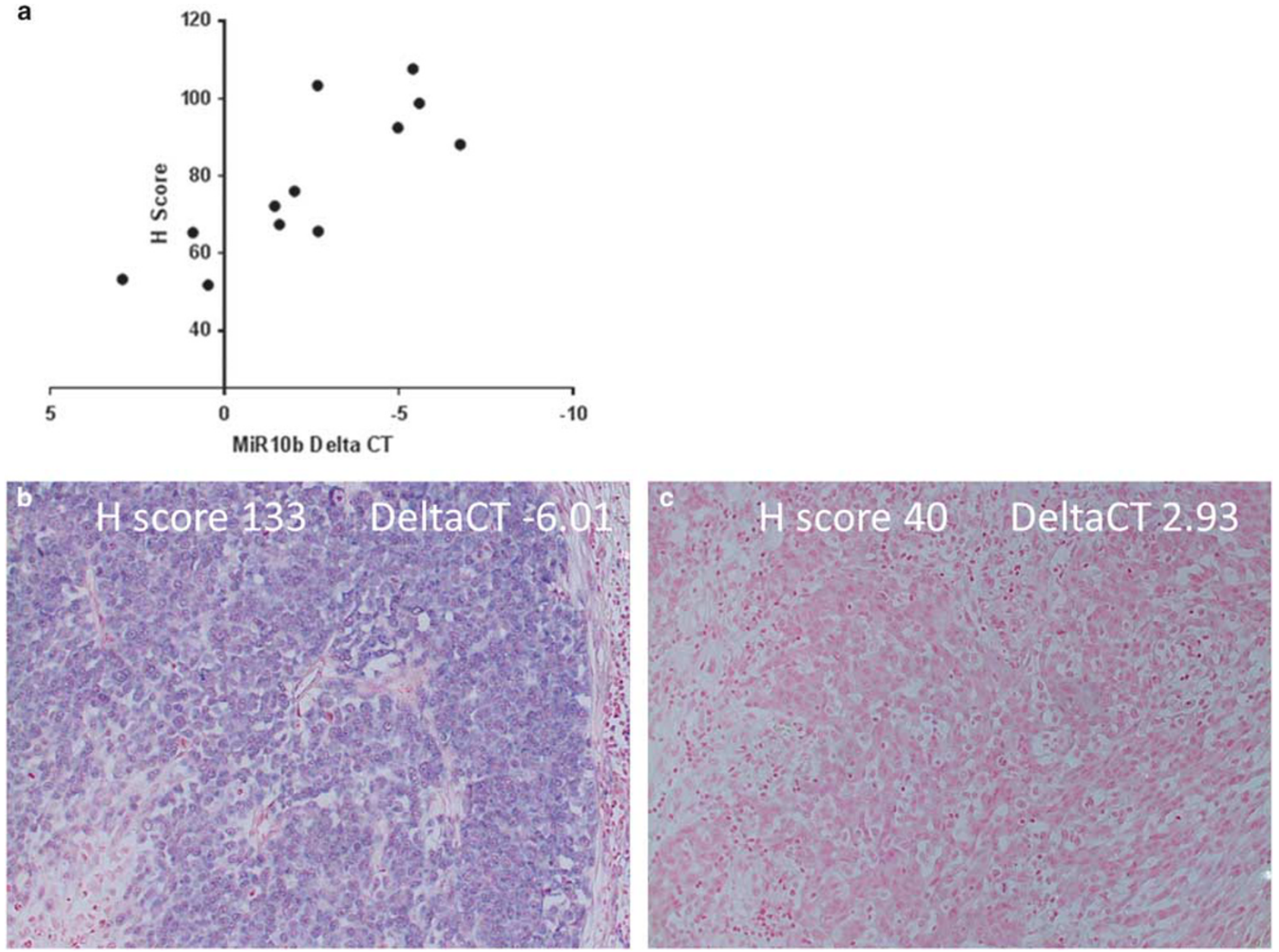

Figure 3 Correlation between miR-10b C-ISH and stem-loop QRT-PCR expression. (a) QRT-PCR expression of miR-10b in 13 melanoma cases correlates with $H$ score in the same tissue section. (b and c) C-ISH of case with high and low miR-10b expression, respectively. Note that the photomicrographs show only an individual image from each of the two cases, whereas the graph shows a mean score from four images per case.

\section{Discussion}

Using class comparison with split-sample validation, we here identify miR-10b as a prognostic miRNA with an ability to distinguish thicker melanomas that metastasized within 5 years from those that did not, suggesting that this miRNA may be useful for identifying aggressive thicker melanomas. We then sought to verify the prognostic value of miR-21, the miR200 family, and miR-205 because these had been studied by us using independent samples previously and also by others. ${ }^{14-26}$ We demonstrate that miR-10b and miR-200b have independent prognostic value in a multivariable logistic regression model, raising the possibility that a miRNA panel could be used to better stratify melanoma patients than AJCC staging alone. Absence of Breslow thickness as an independent prognostic factor from the final multivariable model may reflect the fact that all of these melanomas were relatively thick, suggesting that miRNA expression may have prognostic value in this high-risk melanoma subset. Finally, for miR-10b we show that ISH correlates with QRT-PCR, indicating that ISH could be used in future studies to further validate miR-10b. This data confirms that miR-10b is present in tumor cells and not surrounding stromal elements.

MiR-10b was first identified as a so-called 'oncomir' in breast cancer, ${ }^{33}$ where it enhanced migration and invasion. Several other studies subsequently found increased expression of miR-10b to be associated with aggressive behavior in various cancers. ${ }^{34-38}$ In melanoma, miR-10b was identified in miRNA discovery studies ${ }^{9,39}$ but no studies have focused on miR-10b as the primary melanoma candidate biomarker. Segura et al. ${ }^{9}$ conducted a discovery analysis in melanoma and miR-10b was associated with increased post-recurrence survival, which contradicts our findings. However, this study focused on metastatic tissue and survival after recurrence, whereas our study focuses on the time to first metastasis. Furthermore, the 
considerably more prevalent view of miR-10b is that it is an oncomir. ${ }^{33,40-43}$ The mechanistic role of miR-10b in melanoma was not assessed in this study and remains to be established.

The cases in our study were purposely chosen to include relatively thick tumors, since these have highest risk for disease progression, but this limits the ability to generalize our findings to the wider population of patients with thinner melanomas, but does make the study more relevant to high-risk cases. Discovery studies are beset by the possibility of spurious chance findings due to multiple testing. The fact that none of the miRNAs in the discovery set passed a FDR threshold of $5 \%$ is concerning, but is mitigated in several ways. First, we only

Table 4 miR-200 family, miR205, and miR-21 expression in $\mathrm{P}-\mathrm{M}$ and $\mathrm{P}+\mathrm{M}$ primary melanomas

\begin{tabular}{cccccc}
\hline & $\mathrm{N}$ & Mean $\Delta C_{T}$ & s.d. & $\mathrm{t}(d f=77)$ & $\mathrm{P}$ \\
\hline mir200a & & & & & \\
$\mathrm{P}-\mathrm{M}$ & 40 & 0.00 & 1.51 & & \\
$\mathrm{P}+\mathrm{M}$ & 39 & -2.04 & 5.61 & 2.214 & 0.030 \\
& & & & & \\
mir200b & & & & & \\
$\mathrm{P}-\mathrm{M}$ & 40 & 0.00 & 1.73 & & \\
$\mathrm{P}+\mathrm{M}$ & 39 & -2.80 & 5.67 & 2.981 & 0.004 \\
& & & & & \\
mir200c & & & & & \\
$\mathrm{P}-\mathrm{M}$ & 40 & 0.00 & 1.46 & & \\
$\mathrm{P}+\mathrm{M}$ & 39 & -0.70 & 1.79 & 1.904 & 0.061 \\
mir141 & & & & & \\
$\mathrm{P}-\mathrm{M}$ & 40 & 0.00 & 1.49 & & \\
$\mathrm{P}+\mathrm{M}$ & 39 & -0.81 & 1.94 & 2.077 & 0.041 \\
mir429 & & & & & \\
$\mathrm{P}-\mathrm{M}$ & 40 & 0.00 & 3.57 & & \\
$\mathrm{P}+\mathrm{M}$ & 39 & 0.22 & 3.30 & 0.281 & 0.779 \\
mir205 & & & & & \\
$\mathrm{P}-\mathrm{M}$ & 40 & 0.00 & 1.44 & & \\
$\mathrm{P}+\mathrm{M}$ & 39 & -0.64 & 1.66 & 1.836 & 0.070 \\
MiR21 & & & & & \\
$\mathrm{P}-\mathrm{M}$ & 40 & 0.00 & 1.21 & & \\
$\mathrm{P}+\mathrm{M}$ & 39 & 0.38 & 1.02 & 1.520 & 0.133 \\
\hline & & & & &
\end{tabular}

picked the top-ranked miRNA for further analysis; second, this miRNA passes secondary analytical filters, namely that expression is significantly increased during progression from primary to metastatic melanoma and that the fold change is non-trivial; third, and most importantly we use split-sample validation such that the discovery case set was analyzed entirely separately from a validation case set. Indeed, the whole analysis of the validation set from RNA extraction and beyond occurred only when the discovery set analysis was entirely complete. Unfortunately, we did not have access to mitotic index for several of the melanomas. This is a known prognostic factor but could not be incorporated into the logistic regression analysis. For expediency and rigor, we used an image analysis system to score ISH. However, to make this more translatable to clinical labs it may be better in future to develop a semiquantitative scoring system based on simple visual assessment.

The outlook for patients with advanced melanoma has for many years been bleak because of the lack of effective treatment options. However, recently there has been a transformation in the therapy of this disease with the emergence of targeted treatments and immune checkpoint inhibitors. These treatments can have significant side effects and it is therefore ideal to be able to stratify patients effectively. This becomes even more relevant as therapeutic interest moves toward the adjuvant setting. Identification of prognostic biomarkers has therefore never been more relevant to melanoma care. In this study, we provide evidence that miR-10b may be a prognostic marker and that a panel of miRNAs may add value to AJCC staging. Going forward, larger studies will be required to establish the most-effective panel of miRNAs, determine the suitability of C-ISH, how C-ISH may best be scored and finally to determine a precise prediction algorithm and cutoff for stratification. Finally, it would be useful to have a clearer understanding of the mechanistic role of miR-10b in melanoma. This study shows proof of concept that such further studies are worthwhile.

Table 5 Cox proportional hazards regression

\begin{tabular}{|c|c|c|c|c|c|c|c|}
\hline & $B$ & s.e. & Wald & $d f$ & Significance & $\operatorname{Exp}(B)$ & $95 \% C I$ \\
\hline Age & -0.02 & 0.02 & 0.97 & 1 & 0.324 & 0.98 & $0.94,1.02$ \\
\hline $\begin{array}{l}\text { Gender } \\
\text { Extremity }\end{array}$ & -0.94 & 0.66 & 2.06 & 1 & 0.151 & 0.390 & $0.11,1.41$ \\
\hline Trunk & -0.05 & 0.84 & 0.004 & 1 & 0.952 & 0.95 & $0.18,4.90$ \\
\hline Head and neck & -0.32 & 0.97 & 0.11 & 1 & 0.742 & 0.73 & $0.11,4.83$ \\
\hline Ulceration & -0.51 & 0.60 & 0.70 & 1 & 0.401 & 0.60 & $0.18,1.97$ \\
\hline Depth & 0.24 & 0.15 & 2.59 & 1 & 0.107 & 1.27 & $0.95,1.71$ \\
\hline miR10b & -0.44 & 0.14 & 9.35 & 1 & 0.002 & 0.64 & $0.48,0.85$ \\
\hline mir200b & -0.25 & 0.12 & 3.95 & 1 & 0.047 & 1.28 & $1.00,1.63$ \\
\hline
\end{tabular}




\section{Acknowledgments}

This study was supported by the Hope Against Cancer-Rod Cassady Fellowship.

\section{Disclosure/conflict of interest}

The authors declare no conflict of interest.

\section{References}

1 Bartel DP. microRNAs: genomics, biogenesis, mechanism, and function. Cell 2004;116:281-297.

2 Calin GA, Dumitru CD, Shimizu M et al. Frequent deletions and down-regulation of micro- RNA genes miR15 and miR16 at 13q14 in chronic lymphocytic leukemia. Proc Natl Acad Sci USA 2002;99:15524-15529.

3 Fabbri M. microRNAs and cancer: towards a personalized medicine. Curr Mol Med 2013;13:751-756.

4 Glud M, Klausen M, Gniadecki R et al. microRNA expression in melanocytic nevi: the usefulness of formalin-fixed, paraffin-embedded material for miRNA microarray profiling. J Invest Dermatol 2008;1219-1224.

5 Zhang L, Huang J, Yang $\mathrm{N}$ et al. microRNAs exhibit high frequency genomic alterations in human cancer. Proc Natl Acad Sci USA 2006;103:9136-9141.

6 Gaur A, Jewell DA, Liang Y et al. Characterization of microRNA expression levels and their biological correlates in human cancer cell lines. Cancer Res 2007;67:2456-2468.

7 Caramuta S, Egyhazi S, Rodolfo $\mathrm{M}$ et al. microRNA expression profiles associated with mutational status and survival in malignant melanoma. J Invest Dermatol 2010;130:2062-2070.

8 Satzger I, Mattern A, Kuettler U et al. microRNA-15b represents an independent prognostic parameter and is correlated with tumor cell proliferation and apoptosis in malignant melanoma. Int J Cancer 2010;126: 2553-2562.

9 Segura MF, Belitskaya-Levy I, Rose AE et al. Melanoma microRNA signature predicts post-recurrence survival. Clin Cancer Res 2010;16:1577-1586.

10 Jayawardana K, Schramm SJ, Tembe V et al. Identification, review and systematic cross-validation of microrna prognostic signatures in metastatic melanoma. J Invest Dermatol 2015; doi:10.1038/jid.2015.355 (e-pub ahead of print).

11 Tembe V, Schramm SJ, Stark MS et al. microRNA and mRNA expression profiling in metastatic melanoma reveal associations with BRAF mutation and patient prognosis. Pigment Cell Melanoma Res 2015;28: 254-266.

12 Hanniford D, Zhong J, Koetz L et al. A miRNA-based signature detected in primary melanoma tissue predicts development of brain metastasis. Clin Cancer Res 2015;21:4903-4912.

$13 \mathrm{Li} \mathrm{Z}$, Rana TM. Therapeutic targeting of microRNAs: current status and future challenges. Nat Rev Drug Discov 2014;13:622-638.

14 Saldanha G, Potter L, Shendge $\mathrm{P}$ et al. Plasma microRNA-21 is associated with tumor burden in cutaneous melanoma. J Invest Dermatol 2013;133:1381-1384.

15 Grignol V, Fairchild ET, Zimmerer JM et al. miR-21 and miR-155 are associated with mitotic activity and lesion depth of borderline melanocytic lesions. Br J Cancer 2011;105:1023-1029.

16 Jiang $\mathrm{L}, \mathrm{Lv} \mathrm{X}, \mathrm{Li} \mathrm{J}$ et al. The status of microRNA-21 expression and its clinical significance in human cutaneous malignant melanoma. Acta Histochem 2011;114:582-588.

17 Yang $\mathrm{CH}$, Yue J, Pfeffer SR et al. microRNA-21 regulates the metastatic behavior of B16 melanoma cells. J Biol Chem 2011;286: 39172-39178.

18 Satzger I, Mattern A, Kuettler U et al. microRNA-21 is upregulated in malignant melanoma and influences apoptosis of melanocytic cells. Exp Dermatol 2012;21: 509-514.

19 Martin Del Campo SE, Latchana N, Levine KM et al. MiR-21 enhances melanoma invasiveness via inhibition of tissue inhibitor of metalloproteinases 3 expression: in vivo effects of MiR-21 inhibitor. PLoS One 2015;10:e0115919.

20 Caramel J, Papadogeorgakis E, Hill L et al. A switch in the expression of embryonic EMT-inducers drives the development of malignant melanoma. Cancer Cell 2013;24:466-480.

21 Dar AA, Majid S, de Semir D et al. miRNA-205 suppresses melanoma cell proliferation and induces senescence via regulation of E2F1 protein. J Biol Chem 2011;286:16606-16614.

22 Elson-Schwab I, Lorentzen A, Marshall CJ. microRNA-200 family members differentially regulate morphological plasticity and mode of melanoma cell invasion. PLoS One 2010;5:e13176.

23 Hanna JA, Hahn L, Agarwal S et al. In situ measurement of miR-205 in malignant melanoma tissue supports its role as a tumor suppressor microRNA. Lab Invest 2012;92:1390-1397.

24 Liu S, Tetzlaff MT, Liu A et al. Loss of microRNA-205 expression is associated with melanoma progression. Lab Invest 2012;92:1084-1096.

25 van Kempen LC, van den Hurk K, Lazar V et al. Loss of microRNA-200a and c, and microRNA-203 expression at the invasive front of primary cutaneous melanoma is associated with increased thickness and disease progression. Virchows Arch 2012;461:441-448.

26 Xu Y, Brenn T, Brown ER et al. Differential expression of microRNAs during melanoma progression: miR-200c, miR-205 and miR-211 are downregulated in melanoma and act as tumour suppressors. Br J Cancer 2012;106:553-561.

27 Mestdagh P, Van Vlierberghe P, De Weer A et al. A novel and universal method for microRNA RT-qPCR data normalization. Genome Biol 2009;10:R64.

28 Chen C, Ridzon DA, Broomer AJ et al. Real-time quantification of microRNAs by stem-loop RT-PCR. Nucleic Acids Res 2005;33:e179.

29 Vandesompele J, De Preter K, Pattyn F et al. Accurate normalization of real-time quantitative RT-PCR data by geometric averaging of multiple internal control genes. Genome Biol 2002;3:1-11.

30 Andersen CL, Jensen JL, Orntoft TF. Normalization of real-time quantitative reverse transcription-PCR data: a model-based variance estimation approach to identify genes suited for normalization, applied to bladder and colon cancer data sets. Cancer Res 2004;64:5245-5250.

31 Jorgensen S, Baker A, Moller S et al. Robust one-day in situ hybridization protocol for detection of microRNAs in paraffin samples using LNA probes. Methods 2010;52:375-381. 
32 Benjamini Y, Hochberg Y. Controlling the false discovery rate: a practical and powerful approach to multiple testing. J R Stat Soc Ser B (Methodological) 1995;57:289-300.

33 Ma L, Teruya-Feldstein J, Weinberg RA. Tumour invasion and metastasis initiated by microRNA-10b in breast cancer. Nature 2007;449:682-688.

34 Baffa R, Fassan M, Volinia S et al. microRNA expression profiling of human metastatic cancers identifies cancer gene targets. J Pathol 2009;219:214-221.

35 Garzon R, Garofalo M, Martelli MP et al. Distinctive microRNA signature of acute myeloid leukemia bearing cytoplasmic mutated nucleophosmin. Proc Natl Acad Sci USA 2008;105:3945-3950.

36 Nakata K, Ohuchida K, Mizumoto K et al. microRNA-10b is overexpressed in pancreatic cancer, promotes its invasiveness, and correlates with a poor prognosis. Surgery 2011;150:916-922.

37 Sasayama T, Nishihara $\mathrm{M}$, Kondoh $\mathrm{T}$ et al. microRNA-10b is overexpressed in malignant glioma and associated with tumor invasive factors, uPAR and RhoC. Int J Cancer 2009;125:1407-1413.
38 Tian Y, Luo A, Cai Y et al. microRNA-10b promotes migration and invasion through KLF4 in human esophageal cancer cell lines. J Biol Chem 2010;285: 7986-7994.

39 Jukic DM, Rao UN, Kelly L et al. microRNA profiling analysis of differences between the melanoma of young adults and older adults. J Transl Med 2010;8:27.

40 Chen Y, Stallings RL. Differential patterns of microRNA expression in neuroblastoma are correlated with prognosis, differentiation, and apoptosis. Cancer Res 2007;67:976-983.

41 Guessous F, Alvarado-Velez M, Marcinkiewicz L et al. Oncogenic effects of miR-10b in glioblastoma stem cells. J Neurooncol 2013;112:153-163.

42 Liang S, Chen L, Huang $\mathrm{H}$ et al. The experimental study of miRNA in pituitary adenomas. Turk Neurosurg 2013;23:721-727.

43 Shen Y, Tang D, Yao R et al. microRNA expression profiles associated with survival, disease progression, and response to gefitinib in completely resected nonsmall-cell lung cancer with EGFR mutation. Med Oncol 2013;30:750. 